Published in final edited form as:

Curr Opin Cell Biol. 2007 August ; 19(4): 436-445.

\title{
Signaling on the endocytic pathway
}

\author{
Mark von Zastrow and Alexander Sorkin \\ University of California at San Francisco and University of Colorado Health Sciences Center
}

\section{Summary}

Endocytosis regulates many cellular signaling processes by controlling the number of functional receptors available at the cell surface. Conversely, some signaling processes regulate the endocytic pathway. Furthermore, various cellular signaling events appear to occur on endosome membranes. The endocytic pathway, by providing a set of dynamic and biochemically specialized endomembrane structures that physically communicate with the plasma membrane, is increasingly viewed as a highly flexible scaffold for mediating precise spatiotemporal control and transport of diverse biological signals. General principles of endosome-based signaling are beginning to emerge but, in many cases, the physiological significance of signaling on the endocytic pathway remains poorly understood.

\section{Introduction}

A cardinal feature of biological membranes is their high degree of lateral organization. The plasma membrane, in particular, is remarkably heterogeneous. This heterogeneity is often exploited by signaling receptors, whose segregation in specialized surface domains contributes to efficient and selective transfer of biological information. Such organization is obvious in morphologically differentiated cells of complex tissues, but is not limited to metazoans - or even to eukaryotes. Chemotactic receptors of bacteria, for example, are assembled with related signaling and regulatory proteins in a discrete region of the plasma membrane to facilitate high sensitivity, wide dynamic range and integration of multiple chemical stimuli [1]. The endocytic pathway of eukaryotic cells can be viewed, both topologically and from an evolutionary perspective, as an extension of a heterogeneous plasma membrane. Many signaling receptors traverse the endocytic pathway in a regulated manner, and endosomal membranes are themselves organized into compositionally and functionally specialized domains [2]. It is perhaps not surprising, then, that the endosomal system is intimately involved in cell signaling. Indeed, the available evidence strongly supports such a nexus, to the degree that signaling and endocytic mechanisms have been suggested to represent essentially a single integrated system [3].

Relationships between signaling and endocytosis have been previously reviewed [3-5]. Accordingly, we will briefly summarize earlier work and focus on two areas of recent advance: First, new progress toward understanding how signaling events regulate the endocytic machinery will be summarized. Second, we will focus on present evidence regarding the role of endosomes as signaling organelles. Finally, we will propose some general principles of

\footnotetext{
Adresses: Mark von Zastrow, Departments of Psychiatry and Cellular \& Molecular Pharmacology, N212E Genentech Hall, Box 2140, UCSF Mission Bay Campus, 600 16th Street, San Francisco, CA 94158, phone: 415-476-7855, fax: 415-514-0169, email: mark.vonzastrow@ucsf.edu, Alexander Sorkin, Department of Pharmacology, University of Colorado Health Sciences Center at Fitzsimons, P.O. Box 6511, Mail Stop 8303, Aurora, CO 80045-0508, phone: 303-724-3649, fax: 303-724-3663, email: Alexander.Sorkin@UCHSC.edu

Publisher's Disclaimer: This is a PDF file of an unedited manuscript that has been accepted for publication. As a service to our customers we are providing this early version of the manuscript. The manuscript will undergo copyediting, typesetting, and review of the resulting proof before it is published in its final citable form. Please note that during the production process errors may be discovered which could affect the content, and all legal disclaimers that apply to the journal pertain.
} 
endosome-based signaling and consider the question of whether, and how, such signaling differs from that originating in the plasma membrane.

\section{Endocytosis controls signaling}

Many signaling receptors undergo rapid endocytosis following ligand-induced activation, making endocytosis arguably the most highly conserved process for functional regulation of receptor-mediated signaling from the plasma membrane in eukaryotes (Fig $1 \mathrm{~A})$. While individual signaling receptors differ in their ability to undergo regulated endocytosis, and can selectively enter distinct clathrin -dependent and -independent pathways, the immediate consequence of regulated endocytosis by various mechanisms is to reduce the number of receptors present on the cell surface and thus attenuate cellular responsiveness to extracellular ligand [6].

The subsequent effects of regulated endocytosis on signaling from the plasma membrane can vary considerably, and depend in large part on the sorting of internalized receptors via divergent downstream membrane pathways. Sorting of endocytosed signaling receptors to lysosomes contributes to proteolytic down-regulation of receptor number and typically results in a prolonged attenuation of cellular responsiveness. In many cases lysosomal sorting is promoted by direct ubiquitination of the receptor's cytoplasmic domain, promoting association of receptors with the endosome-associating proteins Hrs/Vps27 and Tsg101/Vps23, which function in cargo delivery to internal membranes of multivesicular late endosomes (MVBs) via a series of endosomal sorting complexes referred to collectively as the "ESCRT" machinery [7,8]. Some signaling receptors, such as opioid neuropeptide receptors, undergo Hrs and ESCRT-dependent sorting to lysosomes in the absence of direct receptor ubiquitination. This process involves distinct, and still poorly understood, non-covalent protein interactions with the receptor [9]. Trafficking of internalized receptors back to the plasma membrane, by contrast, typically restores cellular responsiveness to ligand and occurs for many receptors by "default" -i.e., essentially via bulk membrane flow $[4,7]$.

Certain seven-transmembrane signaling receptors (7TMRs), such as the $\beta_{2}$-adrenergic receptor $\left(\beta_{2} \mathrm{AR}\right)$, do not recycle efficiently by default. A PDZ domain-mediated protein interaction with the $\beta_{2} \mathrm{AR}$ is required to direct recycling via an Hrs-dependent mechanism [10]. One possible reason for such complexity is to promote insertion of receptors into regions of the plasma membrane specialized for particular signaling processes. PDZ-directed recycling of the $\beta_{2} \mathrm{AR}$ in cardiac myocytes has been proposed to "switch" receptor signaling from $\mathrm{G}_{\mathrm{s}}$ to $\mathrm{G}_{\mathrm{i}}$-mediated pathways, organized within distinct surface microdomains, thus producing a biphasic response of myocyte contraction rate to catecholamine activation [11]. Spatially restricted insertion to the plasma membrane has not been directly established in myocytes. Live imaging studies of neurons, however, suggest that $\beta_{2}$ ARs undergo PDZ-directed recycling to surface domains differing in lateral mobility [12].

Endocytosis can also regulate signaling from the plasma membrane by controlling the concentration of ligands as well as other regulatory proteins. Endocytosis of Wingless/Int1 (Wnt) and transforming growth factor- $\beta$ (TGF $\beta$ ) homologue Decapentaplegic (Dpp) ligands, for example, is thought to contribute to shaping long-range gradients important to developing tissues [13]. Hedgehog (Hh) signaling provides an interesting example of signal regulation by endocytic trafficking of the regulatory protein Patched (Ptc), which inhibits the constitutive signaling activity of the seven-transmembrane Smoothened (Smo) protein. Both Ptc and Smo undergo constitutive (ligand-independent) endocytosis at a slow rate. Binding of the Hh ligand is thought to promote sorting of Ptc from Smo in an endosomal compartment, resulting in preferential recycling of Smo and net pathway activation from the plasma membrane [14,15]. 


\section{Signaling controls endocytosis}

The converse regulation - control of the endocytic machinery by signaling events- also occurs (Fig $1 \mathrm{~B}$ ). This is a complementary, rather than contradictory, view that emphasizes the degree to which signaling and trafficking mechanisms are interconnected functionally. A recent study, using a comprehensive RNA interference approach, identified a number of kinases that modulate endocytic processes in cultured human cells; most of the kinases identified function as downstream mediators in conventional receptor-linked signaling pathways. Furthermore, several of the kinases produced opposite effects on clathrin-dependent endocytosis of transferrin compared to clathrin-independent uptake of viruses, suggesting coordinate regulation of distinct endocytic mechanisms [16]. While it is unclear from this study which of these kinases directly influence the endocytic machinery, as opposed to other processes (such as the viral life cycle) required for the assays, activation of p38/MAP kinase induced by stress or cytotoxic drugs has been recently reported to promote internalization of the EGF receptor receptor tyrosine kinase (RTK) by a dual mechanism involving phosphorylation of both the EGF receptor and the endosome-associating Rab5 effector protein EEA.1. By down-regulating EGF receptor survival signaling, p38-mediated internalization enhances the cytotoxic effect of drugs and UV-irradiation [17].

In addition to kinase-linked regulation of endocytosis, which presumably acts globally throughout the cell, recent evidence suggests local regulation of clathrin-coated pit subsets by activated signaling receptors (Fig $1 \mathrm{C}$ ). EGF-dependent formation of coated pits that accumulate EGF receptors, but not transferrin receptors, was revealed in cells depleted of the clathrin adaptor protein AP-2. This effect required activity of the EGF receptor tyrosine kinase, presumably to generate binding sites for the Grb2 adaptor protein [18]. Some 7TMRs have been shown to regulate the dynamics specifically of clathrin-coated pits in which they accumulate. This regulation does not require $\mathrm{G}$ protein-mediated signal transduction, and appears to involve local PDZ domain-dependent linkage of receptors to the cortical actin cytoskeleton [19].

Receptor-mediated signaling can also regulate the endocytic pathway at later stages, downstream of specific endocytic mechanisms operating in the plasma membrane (Fig $1 \mathrm{~B}$ ). Live cell imaging established that maturation of early to late endosomes occurs by dissociation of Rab5 and association of Rab7 with the limiting membrane; signaling via the EGF receptor tyrosine kinase decreased the rate of this Rab "conversion" process, suggesting a specific function of receptor tyrosine kinase signaling in controlling the rate of endosome maturation [20]. On the other hand, studies using quantitative electron microscopy have recently demonstrated that EGF receptor signaling increased the number of MVBs in the cell [21]. A potential concern, which arises in many current studies, is that regulatory effects were typically demonstrated in cells that highly overexpress signaling receptors. It remains to be determined, therefore, whether particular regulatory effects on the endocytic machinery are significant under physiological conditions.

\section{Signaling from the endosome membrane}

In addition to extensive and converse interactions between the endocytic pathway and plasma membrane-delimited signaling events, endosome membranes are themselves important sites of receptor-initiated signal transduction. Several examples are discussed below and diagrammed in Figure 2.

\section{RTK signaling and the concept of "signaling endosomes"}

The hypothesis of signaling from endosomes originated from biochemical fractionation of RTK signaling components [22]. The key finding was the presence in endosomes of activated EGF 
receptors, as well as various downstream effectors such as components of the Ras activation pathway. For example, Grb2, Shc and SOS remain associated with EGF receptors in endosomes, thus allowing activation of Ras, particularly the H-Ras form that is located on the same endosomes [22,23]. The RTK signaling endosome hypothesis has been further refined and supported in many subsequent studies [4,24]. In view of increasingly compelling evidence for colocalization and interaction of various RTK signaling components on the endosome membrane, it is presently difficult to imagine how signal transduction would not occur from the endosome membrane. A more challenging question is to understand the specific physiological significance of signaling from the endosome membrane. Are endosome-based signals functionally distinct from those emanating from the plasma membrane, or do endosome-based events represent a spatial and/or temporal extension of a signal that is otherwise identical to that initiated at the cell surface?

Early efforts to address this question, using inhibition of endocytosis by mutant dynamin, suggested that EGF signaling from endosomes produces a distinct spectrum of downstream kinase activation [25]. Subsequent studies showing pleiotropic effects of mutant dynamin expression, however, suggest caution in this interpretation (discussed in [4]). Endocytic inhibition by RNAi-mediated depletion of clathrin suggested, more recently, that endocytosis is required for full activation of ERK1/2 by the VEGF receptor [26]. Taken together, and despite caveats, such studies suggest an important function of endocytosis in mediating the full biological activity of RTK activation. They do not, however, resolve whether the signaling effects of endocytosis are qualitative or quantitative in nature.

Perhaps the strongest evidence for a qualitatively distinct, and physiologically important, function of endosome-based signaling from RTKs comes from the study of signaling by the TrkA neurotrophin receptor. A number of studies, using various approaches ranging from subcellular fractionation of receptor-containing endosomes to manipulation of signaling and trafficking in compartmentalized cell culture, support the hypothesis that TrkA-containing endosomes contribute to retrograde transport of important survival signals (reviewed in [27]). Endosomes containing activated TrkA complexes selectively mediate prolonged activation of Rap1, whereas receptors present in the plasma membrane transiently activate Ras [28]. Retrograde transport of signaling endosomes is thought to promote survival via a pathway involving ERK5 activation and CREB-dependent transcription in the soma, whereas TrkA signaling in axonal tips activates only ERK1/2 locally [29]. Endocytosis can also, however, promote ubiquitination-dependent down-regulation of TrkA and reduced strength of survival signaling [30]. Thus, endocytosis appears to produce both qualitative and quantitative effects on TrkA signaling.

Another question regarding the specificity of endosome-based signaling is the degree to which endosomes engaged in signaling are specialized. Are there specific endosomes dedicated to signaling but not trafficking functions? The experimental findings pertinent to this question are presently mixed. For example, one argument in favor of specialized signaling endosomes came from the observation that the putative transcription factor APPL, which undergoes nuclear translocation in response to EGF receptor activation, localizes to a population of endosomes that associate with Rab5 but do not contain the resident early endosomal protein EEA.1 [31]. Recently, however, APPL-associated endosomes were found to be EEA.1-positive in PC12 cells, and APPL was proposed to mediate a distinct function in endocytic trafficking of TrkA involving APPL interaction with the endosome-localizing PDZ protein GIPC [32]. Other recent studies reveal the involvement of late endosomal protein Rab7 in the retrograde transport of TrkA [33]. It is likely that a sorting step occurs before the long-distance retrograde transport of signaling components in Rab7-containing endosomes. This step may allow enrichment of a subset of specialized signaling endosomes in TrkA and associated proteins, at the expense of resident proteins of early endosomes and itinerant proteins such as nutrient 
receptors, although the degree to which such TrkA-containing endosomes differ from other Rab7-positive late endosomes is presently not known.

\section{Evidence for endosome signaling elicited by 7TMR activation}

Various 7TMRs, historically called G protein-coupled receptors (GPCRs) because of their ability to signal via heterotrimeric $\mathrm{G}$ proteins in the plasma membrane, may mediate distinct modes of signal transduction from endocytic membranes. Early studies supporting this hypothesis, and their caveats, have been discussed previously [4]. A major mechanism by which mammalian 7TMRs are presently thought to initiate endosome-based signaling is via interaction with arrestins. Arrestins, first recognized for their ability to bind to activated 7TMRs and "arrest" signaling via heterotrimeric G proteins, can mediate at least two additional cellular functions. The main arrestins expressed outside the visual system, arrestins 2 and 3 (also called $\beta$-arrestin- 1 and -2 , respectively) function as regulated endocytic adaptors for 7TMRs by binding directly to clathrin, AP-2, and PIP2 and by undergoing regulated ubiquitination that further promotes coated pit association. Second, these arrestins bind various components of MAP kinase modules (particularly MAPKKK and MAPK enzymes), and thus appear to serve as scaffolds for MAP kinase signaling [34,35].

Some 7TMRs robustly recruit arrestins to the endosome membrane, and colocalize in endosomes with activated MAP kinase components, supporting a signaling endosome hypothesis analogous to that proposed previously for RTKs. A number of other 7TMRs dissociate from arrestins upon endocytosis and promote MAP kinase activation less strongly or for a shorter duration [36]. Binding of activated MAP kinases to endosomes may also restrict enzyme access to nuclear effectors [36,37]. Accordingly, the present evidence suggests the existence of both quantitative and qualitative differences in endosome-based signaling via arrestins.

Endosome-based signaling is also thought to occur from more distantly related 7TMRs such as Frizzled (Fzl), sometimes called an atypical 7TMR because it has not been shown clearly to signal via heterotrimeric G proteins. Activation of Fzl by secreted Wnt ligands induces endocytosis of Fzl/Wnt/Arrow complexes via clathrin-coated pits. The receptor-associated protein Dishevelled (Dvl) promotes endocytosis of the complex by binding directly to AP-2, which appears to be essential for signaling of Fzl via Dvl [38-40]. While Dvl can also interact with arrestins when overexpressed in cultured mammalian cells [41], the importance of this interaction for Fzl endocytosis or signaling in vivo has been questioned [40].

An interesting additional elaboration of the signaling endosome hypothesis was suggested recently from study of the mating pheromone response pathway in the budding yeast $S$. cerevisiae. The secreted $\alpha$-factor mediates its cellular effects by activating the 7TMR Ste2 present in the plasma membrane. Yeast do not express arrestins, and all known signaling from Ste2 is dependent on heterotrimeric G protein activation. Previous studies indicated that the mating pheromone signal is transduced by the plasma membrane-delimited $\beta \gamma$ subcomplex of the heterotrimeric $\mathrm{G}$ protein upon receptor-promoted dissociation from the $\alpha$ subunit (Gpa1). A mutationally activated form of Gpa1 localized to endosomes, mediated $\beta \gamma$-independent activation of the endosome-localized Vps34 PI3 kinase by direct interaction, and promoted endosome recruitment of the FYVE domain-containing signaling adaptor Bem1. Deletion of either VPS34 or VPS15 (Vps15 associates on the endosome membrane with Vps34 and is required for full PI3K activity) reduced the strength and duration of receptor-mediated pheromone response mediated by the Fus3 MAP kinase [42]. This finding places endosomedelimited PI3K in the natural pheromone response pathway and identifies a novel type of G protein-dependent signaling that can occur from endosomes. 


\section{Delta / Notch and proteolytic signaling from endosomes}

Another extension of the signaling endosome hypothesis involves signal transduction mediated by proteolysis occurring in the endosome membrane, which was discovered in studies of cell fate decisions controlled by Notch receptors. Notch receptors are dimeric integral membrane proteins that require two sequential proteolytic cleavages for their activity in a signal-receiving cell. Notch is activated by direct contact with a signal-sending cell via engagement of a surfaceassociated DSL ligand (including Delta, Serrate, Lag-2, and Jagged) expressed on the signalsending cell [43]. DSL ligand engagement promotes proteolytic cleavage of the ligand-binding ectodomain of Notch (mediated by an ADAM-family protease associated with the extrafacial surface of the receiving cell) followed by a second endoproteolytic cleavage (mediated by an intramembrane aspartyl protease complex called $\gamma$-secretase), which produces a cytoplasmic fragment functioning as a transcriptional regulator. The $\gamma$-secretase complex is present in intracellular compartments including endosomes, potentially explaining the endocytic requirement for Notch signaling. Dynamin-dependent endocytosis is also required for activity of the DSL ligand on the signal-sending cell, possibly due to another proteolytic step required to activate the ligand [43].

An increasing number of $\gamma$-secretase substrates have been identified, including p75 neurotrophin receptors, for which endoproteolytic cleavage has been reported to occur in endosomes after NGF-induced endocytosis [44]. Thus endoproteolytic cleavage, like phosphorylation, may represent a general type of post-translational modification contributing to cell signaling from endosomes. It is interesting that the $\gamma$-secretase complex was independently identified based on its ability to mediate amyloid $\beta$-peptide production from amyloid precursor protein (APP) associated with Alzheimer's disease [45]. A recent study found that amyloid $\beta$-peptide production is stimulated by activation of the $\beta_{2} \mathrm{AR}$, and that this regulation requires endocytosis the receptor, suggesting a potential role of 7TMRs in proteolytic signaling from endosomes and in promoting an important human pathophysiology [46].

\section{Other recent evidence for endosome-based signaling}

The concept of signaling endosomes has been extended to various other receptor systems. For example TGF $\beta$ signaling has been proposed to occur from endosomes via receptor interaction with the FYVE domain-containing protein SARA, which is localized at steady state to the endosome membrane and also binds Smad2/3 proteins that subsequently translocate to the nucleus [47]. Interestingly, interaction of the TGF $\beta$ receptor with another FYVE-domaincontaining protein, endofin, promotes phosphorylation and nuclear translocation of Smad4 [48]. Thus specific scaffolding interactions of TGF $\beta$ receptors in endosomes may trigger distinct signaling responses.

Internalization of tumor necrosis factor-1 receptor and CD95 was implicated in activation of signaling pathways driving programmed cell death [49,50]. Recruitment of death-inducing signaling complexes (DISCs), which promote autoproteolytic cleavage of caspase-8 leading to apoptosis, appears to occur mainly in endosomes. Complexes of Toll-like receptor 9, adaptor protein MyD88 and IRF-7 transcription factor were found in endosomes of plasmacytoid dendritic cells [51]; retention of these receptor complexes in early endosomes was necessary and sufficient for robust interferon induction. Toll-like receptor 3 triggers anti-viral immune response in a ligand (double-stranded RNA)-dependent manner by recruiting and activating cSrc kinase in endosomes of monocyte-derived dendritic cells [52]. Together, these studies implicate endosomal signaling in innate immune responses to viral and bacterial infection.

Moreover, late endosomes have been proposed to serve as a site of assembly of signaling complexes that do not contain receptors. MAP kinase scaffold protein MP1, capable of binding 
to MEK1 and ERK1/2, is associated with the late endosomal protein p14 [53]. Knockdown of p14 in human cells and knockout of p14 in mouse demonstrated the importance of this protein for full activation of ERK1/2 [53,54]. However, mouse knockout experiments have also revealed that, in addition to its function in MAPK signaling, p14 has a role in late endosome biogenesis and receptor sorting [54]. To our knowledge it is presently unclear if this endocytic function of p14 is interrelated with, or distinct from, its MAPK scaffolding function.

\section{Conclusions}

The hypothesis that signaling and the endocytic pathway are intimately linked has continued to gather experimental support during the past several years, and substantial advances have been made toward elucidating this relationship on two fronts. First, receptor-mediated signaling events specifically regulate the endocytic pathway, both globally via activation of various protein kinases and locally by direct protein interactions with individual receptors. Second, increasingly strong evidence implicates endosomes as sites of functional signal transduction. The concept of the signaling endosome, proposed initially based on biochemical fractionation of RTK signaling components from hepatocytes, has been generalized to a wide variety of cell types and now represents a rather mainstream -and even fashionable- concept being considered for diverse signaling systems.

Several principles of endosome-based signaling emerge from synthesis of the presently available data. First, and most generally, to our knowledge all reported examples of endosomal signaling involve some form of induced proximity. Endosome membranes are ideally suited to act as a specialized signaling platform, with distinct biochemical composition (such as enrichment in phosphatidylinositol 3-phosphate) that promotes selective recruitment of various scaffold proteins and signaling mediators. Second, because endosomal membranes communicate with but are not directly exposed to the extracellular milieu, endosome-based signaling can prolong certain signals beyond the duration of ligand exposure at the cell surface, contributing a "memory" function to integrate biological signals. Third, endosomes can function in some cells as vehicles for physical transport of a biological signal, as argued most convincingly by studies of TrkA-mediated retrograde neurotrophin signaling.

Despite the accelerating pace of research on endosomal signaling in diverse systems, many interesting questions remain. Foremost among these, in many cases, is the question of physiological significance. Do endosome-based signals differ fundamentally from those occurring from the plasma membrane and, if so, how? The available evidence suggests a complex answer, depending on the signaling system under consideration. Some examples provide strong evidence for qualitatively distinct endosome-based signaling effects. Most notably, endosome-based signaling by TrkA involves both distinct biochemical signals (Rap1, ERK5) and long-range physical transport from the site of endocytosis in the axon to nuclear effectors in the cell body. In other examples, such as signaling by EGF receptors and 7TMRs, current evidence supports both qualitative and quantitative distinctions between signaling from the plasma membrane and endosomes. Even TGF $\beta$ signaling via Smads, although facilitated by endosome-localized SARA, can also occur to some degree from the plasma membrane $[47,55,56]$. Furthermore, the $\gamma$-secretase complex required for Notch signaling is not restricted to endosomes, and may be catalytically active also in the plasma membrane [57]. Thus it appears likely that signaling from endosomes can involve events that are biochemically distinct from those elicited from the plasma membrane, as well as similar events differing in strength, duration or location.

Future studies will thus require more precise evaluation of both qualitative and quantitative effects of endocytosis on cellular signaling, as well as enhanced spatiotemporal resolution of signaling events occurring on distinct subcellular compartments. Computational models of 
signaling networks are likely to provide important help in analyzing complex spatiotemporal data and predicting functional outcomes under physiological conditions (e.g., [58,59]). An important related goal, given that much of the current evidence on endosome-based signaling mechanisms is derived from heterologous cell models that over-express critical proteins, is to carry out further mechanistic studies using native or near-native cell and tissue preparations. Another exciting challenge is to develop increasingly specific manipulations of mechanisms that mediate or regulate endocytic trafficking, which can be applied acutely and with reduced pleiotropic effects that confound much of the present functional data on endosome-based signaling. Chemical approaches to the latter problem (e.g., $[60,61]$ ), in addition to providing improved tools to facilitate definitive mechanistic and functional analysis, may identify new leads for possible therapeutic exploration.

\section{Acknowledgements}

Given the constraints on text length and reference number, we regret that we were not able to discuss or cite all of the important work underlying the conclusions discussed in this review. We thank our many colleagues for valuable discussion, insights, and criticism. Drs. Henry Bourne, Emilia Galperin, Aylin Hanyaloglu and Manojkumar Puthenveedu provided critical comments on the manuscript. We also gratefully acknowledge the National Institutes of Health, American Cancer Society, American Heart Association, and the National Alliance for Research on Schizophrenia and Depression (NARSAD) for supporting research and training in our laboratories.

\section{References}

1. Sourjik V. Receptor clustering and signal processing in E. coli chemotaxis. Trends Microbiol 2004;12:569-576. [PubMed: 15539117]

2. Gruenberg J. The endocytic pathway: a mosaic of domains. Nat Rev Mol Cell Biol 2001;2:721-730. [PubMed: 11584299]

3. Polo S, Di Fiore PP. Endocytosis conducts the cell signaling orchestra. Cell 2006;124:897-900. [PubMed: 16530038]

4. Sorkin A, Von Zastrow M. Signal transduction and endocytosis: close encounters of many kinds. Nat Rev Mol Cell Biol 2002;3:600-614. [PubMed: 12154371]

5. Miaczynska M, Pelkmans L, Zerial M. Not just a sink: endosomes in control of signal transduction. Curr Opin Cell Biol 2004;16:400-406. [PubMed: 15261672]

6. Tsao PI, von Zastrow M. Diversity and specificity in the regulated endocytic membrane trafficking of G-protein-coupled receptors. Pharmacol Ther 2001;89:139-147. [PubMed: 11316517]

7. Gruenberg J, Stenmark H. The biogenesis of multivesicular endosomes. Nat Rev Mol Cell Biol 2004;5:317-323. [PubMed: 15071556]

8. Hurley JH, Emr SD. The ESCRT complexes: structure and mechanism of a membrane-trafficking network. Annu Rev Biophys Biomol Struct 2006;35:277-298. [PubMed: 16689637]

9. Hislop JN, Marley A, Von Zastrow M. Role of mammalian VPS proteins in endocytic trafficking of a non-ubiquitinated $\mathrm{G}$ protein-coupled receptor to lysosomes. J Biol Chem. 2004

10. Hanyaloglu AC, McCullagh E, von Zastrow M. Essential role of Hrs in a recycling mechanism mediating functional resensitization of cell signaling. Embo J 2005;24:2265-2283. [PubMed: 15944737]

11. Xiang Y, Kobilka BK. Myocyte adrenoceptor signaling pathways. Science 2003;300:1530-1532. [PubMed: 12791980]

12. Yudowski GA, Puthenveedu MA, von Zastrow M. Distinct modes of regulated receptor insertion to the somatodendritic plasma membrane. Nat Neurosci 2006;9:622-627. [PubMed: 16604070]

13. Gonzalez-Gaitan M. Signal dispersal and transduction through the endocytic pathway. Nat Rev Mol Cell Biol 2003;4:213-224. [PubMed: 12612640]

14. Incardona JP, Gruenberg J, Roelink H. Sonic hedgehog induces the segregation of patched and smoothened in endosomes. Curr Biol 2002;12:983-995. [PubMed: 12123571]

15. Piddini E, Vincent JP. Modulation of developmental signals by endocytosis: different means and many ends. Curr Opin Cell Biol 2003;15:474-481. [PubMed: 12892789] 
16. Pelkmans L, Fava E, Grabner H, Hannus M, Habermann B, Krausz E, Zerial M. Genome-wide analysis of human kinases in clathrin- and caveolae/raft-mediated endocytosis. Nature 2005;436:78-86. [PubMed: 15889048]

17. Zwang Y, Yarden Y. p38 MAP kinase mediates stress-induced internalization of EGFR: implications for cancer chemotherapy. Embo J 2006;25:4195-4206. [PubMed: 16932740]

18. Johannessen LE, Pedersen NM, Pedersen KW, Madshus IH, Stang E. Activation of the epidermal growth factor (EGF) receptor induces formation of EGF receptor- and Grb2-containing clathrincoated pits. Mol Cell Biol 2006;26:389-401. [PubMed: 16382132]

19. Puthenveedu MA, von Zastrow M. Cargo regulates clathrin-coated pit dynamics. Cell 2006;127:113124. [PubMed: 17018281]

20. Rink J, Ghigo E, Kalaidzidis Y, Zerial M. Rab conversion as a mechanism of progression from early to late endosomes. Cell 2005;122:735-749. [PubMed: 16143105]

21. White IJ, Bailey LM, Aghakhani MR, Moss SE, Futter CE. EGF stimulates annexin 1-dependent inward vesiculation in a multivesicular endosome subpopulation. Embo J 2006;25:1-12. [PubMed: 16052208]

22. Di Guglielmo GM, Baass PC, Ou WJ, Posner BI, Bergeron JJ. Compartmentalization of SHC, GRB2 and $\mathrm{mSOS}$, and hyperphosphorylation of Raf-1 by EGF but not insulin in liver parenchyma. Embo J 1994;13:4269-4277. [PubMed: 7925272]

23. Jiang X, Sorkin A. Coordinated traffic of Grb2 and Ras during epidermal growth factor receptor endocytosis visualized in living cells. Mol Biol Cell 2002;13:1522-1535. [PubMed: 12006650]

24. Li HS, Stolz DB, Romero G. Characterization of endocytic vesicles using magnetic microbeads coated with signalling ligands. Traffic 2005;6:324-334. [PubMed: 15752137]

25. Vieira AV, Lamaze C, Schmid SL. Control of EGF receptor signaling by clathrin-mediated endocytosis. Science 1996;274:2086-2089. [PubMed: 8953040]

26. Lampugnani MG, Orsenigo F, Gagliani MC, Tacchetti C, Dejana E. Vascular endothelial cadherin controls VEGFR-2 internalization and signaling from intracellular compartments. J Cell Biol 2006;174:593-604. [PubMed: 16893970]

27. Zweifel LS, Kuruvilla R, Ginty DD. Functions and mechanisms of retrograde neurotrophin signalling. Nat Rev Neurosci 2005;6:615-625. [PubMed: 16062170]

28. Wu C, Lai CF, Mobley WC. Nerve growth factor activates persistent Rap1 signaling in endosomes. J Neurosci 2001;21:5406-5416. [PubMed: 11466412]

29. Watson FL, Heerssen HM, Bhattacharyya A, Klesse L, Lin MZ, Segal RA. Neurotrophins use the Erk5 pathway to mediate a retrograde survival response. Nat Neurosci 2001;4:981-988. [PubMed: 11544482]

30. Arevalo JC, Waite J, Rajagopal R, Beyna M, Chen ZY, Lee FS, Chao MV. Cell survival through Trk neurotrophin receptors is differentially regulated by ubiquitination. Neuron 2006;50:549-559. [PubMed: 16701206]

31. Miaczynska M, Christoforidis S, Giner A, Shevchenko A, Uttenweiler-Joseph S, Habermann B, Wilm M, Parton RG, Zerial M. APPL proteins link Rab5 to nuclear signal transduction via an endosomal compartment. Cell 2004;116:445-456. [PubMed: 15016378]

32. Varsano T, Dong MQ, Niesman I, Gacula H, Lou X, Ma T, Testa JR, Yates JR 3rd, Farquhar MG. GIPC is recruited by APPL to peripheral TrkA endosomes and regulates TrkA trafficking and signaling. Mol Cell Biol 2006;26:8942-8952. [PubMed: 17015470]

-33. Deinhardt K, Salinas S, Verastegui C, Watson R, Worth D, Hanrahan S, Bucci C, Schiavo G. Rab5 and Rab7 control endocytic sorting along the axonal retrograde transport pathway. Neuron 2006;52:293-305. [PubMed: 17046692] This study used purification strategy based on a fragment of tetanus neurotoxin (TeNT HC) conjugated to paramagnetic beads to identify Rab7 as a functional marker of a specific pool of axonal retrograde carriers, which transport neurotrophins and their receptors, such as p75NTR, TrkB, and BDNF, from the neuromuscular junction with the motor neuron soma. It is shown that Rab5 is essential for an early step in TeNT HC sorting but is absent from axonally transported vesicles. In contrast, Rab7 plays an essential role in axonal retrograde transport of these vesicles

34. Lefkowitz RJ, Shenoy SK. Transduction of receptor signals by beta-arrestins. Science 2005;308:512517. [PubMed: 15845844] 
35. Moore CA, Milano SK, Benovic JL. Regulation of Receptor Trafficking by GRKs and Arrestins. Annu Rev Physiol. 2006

36. Tohgo A, Choy EW, Gesty-Palmer D, Pierce KL, Laporte S, Oakley RH, Caron MG, Lefkowitz RJ, Luttrell LM. The stability of the G protein-coupled receptor-beta-arrestin interaction determines the mechanism and functional consequence of ERK activation. J Biol Chem 2003;278:6258-6267. [PubMed: 12473660]

-37. Ahn S, Shenoy SK, Wei H, Lefkowitz RJ. Differential kinetic and spatial patterns of beta-arrestin and $\mathrm{G}$ protein-mediated ERK activation by the angiotensin II receptor. J Biol Chem 2004;279:35518-35525. [PubMed: 15205453]Chemical inhibitors and RNA interference were used to resolve two components of ERK1/2 activation induced by activation of angiotensin II type $1 \mathrm{~A}$ receptors, a 7TMR. An early and transient ERK signal was dependent on the heterotrimeric $\mathrm{G}$ protein $(\mathrm{s}) \mathrm{G}_{\mathrm{q} / 11}$, and a sustained phase of ERK signaling did not require $\mathrm{G}$ proteins and was inhibited by knockdown of arrestin 3 ( $\beta$-arrestin-2) expression. ERK phosphorylated by the G proteindependent mechanism was localized in the cytoplasm and nucleus, whereas ERK phosphorylated by the arrestin-dependent mechanism localized to endosomes and was excluded from the nucleus

38. Seto ES, Bellen HJ. Internalization is required for proper Wingless signaling in Drosophila melanogaster. J Cell Biol 2006;173:95-106. [PubMed: 16606693]

39. Blitzer JT, Nusse R. A critical role for endocytosis in Wnt signaling. BMC Cell Biol 2006;7:28. [PubMed: 16824228]

-40. Yu A, Rual JF, Tamai K, Harada Y, Vidal M, He X, Kirchhausen T. Association of dishevelled with the clathrin ap-2 adaptor is required for frizzled endocytosis and planar cell polarity signaling. Dev Cell 2007;12:129-141. [PubMed: 17199046]A yeast two-hybrid screen identified Dvl2 interacting with the $\mu 2$-subunit of AP-2, which required both the DEP domain and a tyrosine-containing (YHEL) sequence in Dvl2. Mutationof the YHEL sequence inhibited Wnt5A-induced endocytosis of the atypical 7TMR Fzl4 and selectively interfered with non-canonical ( $\square$-catenin-independent) Wnt signaling prequired for dorsal-ventral axis formation in Xenopus embryos. This study implicates Dvl-mediated association with AP-2 in promoting Fzl endocytosis and mediating Wnt signaling via the planar cell polarity $(\mathrm{PCP})$ pathway

41. Chen W, ten Berge D, Brown J, Ahn S, Hu LA, Miller WE, Caron MG, Barak LS, Nusse R, Lefkowitz RJ. Dishevelled 2 recruits beta-arrestin 2 to mediate Wnt5A-stimulated endocytosis of Frizzled 4. Science 2003;301:1391-1394. [PubMed: 12958364]

••42. Slessareva JE, Routt SM, Temple B, Bankaitis VA, Dohlman HG. Activation of the phosphatidylinositol 3-kinase Vps34 by a G protein alpha subunit at the endosome. Cell 2006;126:191-203. [PubMed: 16839886]An activated mutant G protein $\alpha$-subunit Gpa1 and a genedeletion strain library were used to demonstrate a requirement for VPS34 and VPS15 in mediating the full mating response. Gpa1 was found to bind directly to Vps15 on the endosome membrane, and Vps15 was proposed to function on the endosome membrane as a structural homologue of the $\mathrm{G}$ protein $\beta \gamma$ subunit, which is embedded in the plasma membrane and mediates plasma membranedelimited signaling elicited by $\alpha$-factor-mediated activation of the 7TMR Ste2. This study provides direct evidence for $\mathrm{G}$ protein dependent signaling from endosomes

43. Le Borgne R, Bardin A, Schweisguth F. The roles of receptor and ligand endocytosis in regulating Notch signaling. Development 2005;132:1751-1762. [PubMed: 15790962]

44. Urra MS, Escudero CA, Ramos P, Lisbona MF, Allende E, Covarrubias P, Parraguez JI, Zampieri $\mathrm{N}$, Chao MV, Annaert W, et al. TrkA receptor activation by NGF induces shedding of the p75 neurotrophin receptor followed by endosomal gamma-secretase-mediated release of the p75 intracellular domain. J Biol Chem. 2007

45. Wolfe MS, Xia W, Ostaszewski BL, Diehl TS, Kimberly WT, Selkoe DJ. Two transmembrane aspartates in presenilin-1 required for presenilin endoproteolysis and gamma-secretase activity. Nature 1999;398:513-517. [PubMed: 10206644]

46. Ni Y, Zhao X, Bao G, Zou L, Teng L, Wang Z, Song M, Xiong J, Bai Y, Pei G. Activation of beta2adrenergic receptor stimulates gamma-secretase activity and accelerates amyloid plaque formation. Nat Med 2006;12:1390-1396. [PubMed: 17115048]

47. Di Guglielmo GM, Le Roy C, Goodfellow AF, Wrana JL. Distinct endocytic pathways regulate TGFbeta receptor signalling and turnover. Nat Cell Biol 2003;5:410-421. [PubMed: 12717440] 
48. Chen YG, Wang Z, Ma J, Zhang L, Lu Z. Endofin, a FYVE domain protein, interacts with Smad4 and facilitates TGF-beta signaling. J Biol Chem. 2007

49. Schneider-Brachert W, Tchikov V, Neumeyer J, Jakob M, Winoto-Morbach S, Held-Feindt J, Heinrich M, Merkel O, Ehrenschwender M, Adam D, et al. Compartmentalization of TNF receptor 1 signaling: internalized TNF receptosomes as death signaling vesicles. Immunity 2004;21:415-428. [PubMed: 15357952]

50. Lee KH, Feig C, Tchikov V, Schickel R, Hallas C, Schutze S, Peter ME, Chan AC. The role of receptor internalization in CD95 signaling. Embo J 2006;25:1009-1023. [PubMed: 16498403]

51. Honda K, Ohba Y, Yanai H, Negishi H, Mizutani T, Takaoka A, Taya C, Taniguchi T. Spatiotemporal regulation of MyD88-IRF-7 signalling for robust type-I interferon induction. Nature 2005;434:10351040. [PubMed: 15815647]

52. Johnsen IB, Nguyen TT, Ringdal M, Tryggestad AM, Bakke O, Lien E, Espevik T, Anthonsen MW. Toll-like receptor 3 associates with c-Src tyrosine kinase on endosomes to initiate antiviral signaling. Embo J 2006;25:3335-3346. [PubMed: 16858407]

53. Teis D, Wunderlich W, Huber LA. Localization of the MP1-MAPK scaffold complex to endosomes is mediated by p14 and required for signal transduction. Dev Cell 2002;3:803-814. [PubMed: 12479806]

54. Teis D, Taub N, Kurzbauer R, Hilber D, de Araujo ME, Erlacher M, Offterdinger M, Villunger A, Geley S, Bohn G, et al. p14-MP1-MEK1 signaling regulates endosomal traffic and cellular proliferation during tissue homeostasis. J Cell Biol 2006;175:861-868. [PubMed: 17178906]

55. Lu Z, Murray JT, Luo W, Li H, Wu X, Xu H, Backer JM, Chen YG. Transforming growth factor beta activates Smad2 in the absence of receptor endocytosis. J Biol Chem 2002;277:29363-29368. [PubMed: 12034739]

56. Shi Y, Massague J. Mechanisms of TGF-beta signaling from cell membrane to the nucleus. Cell 2003;113:685-700. [PubMed: 12809600]

57. Chyung JH, Raper DM, Selkoe DJ. Gamma-secretase exists on the plasma membrane as an intact complex that accepts substrates and effects intramembrane cleavage. J Biol Chem 2005;280:43834392. [PubMed: 15569674]

58. Kholodenko BN. Cell-signalling dynamics in time and space. Nat Rev Mol Cell Biol 2006;7:165176. [PubMed: 16482094]

59. Liu G, Swihart MT, Neelamegham S. Sensitivity, principal component and flux analysis applied to signal transduction: the case of epidermal growth factor mediated signaling. Bioinformatics 2005;21:1194-1202. [PubMed: 15531606]

60. Kenski DM, Zhang C, von Zastrow M, Shokat KM. Chemical genetic engineering of G proteincoupled receptor kinase 2. J Biol Chem 2005;280:35051-35061. [PubMed: 16081410]

61. Macia E, Ehrlich M, Massol R, Boucrot E, Brunner C, Kirchhausen T. Dynasore, a cell-permeable inhibitor of dynamin. Dev Cell 2006;10:839-850. [PubMed: 16740485] 


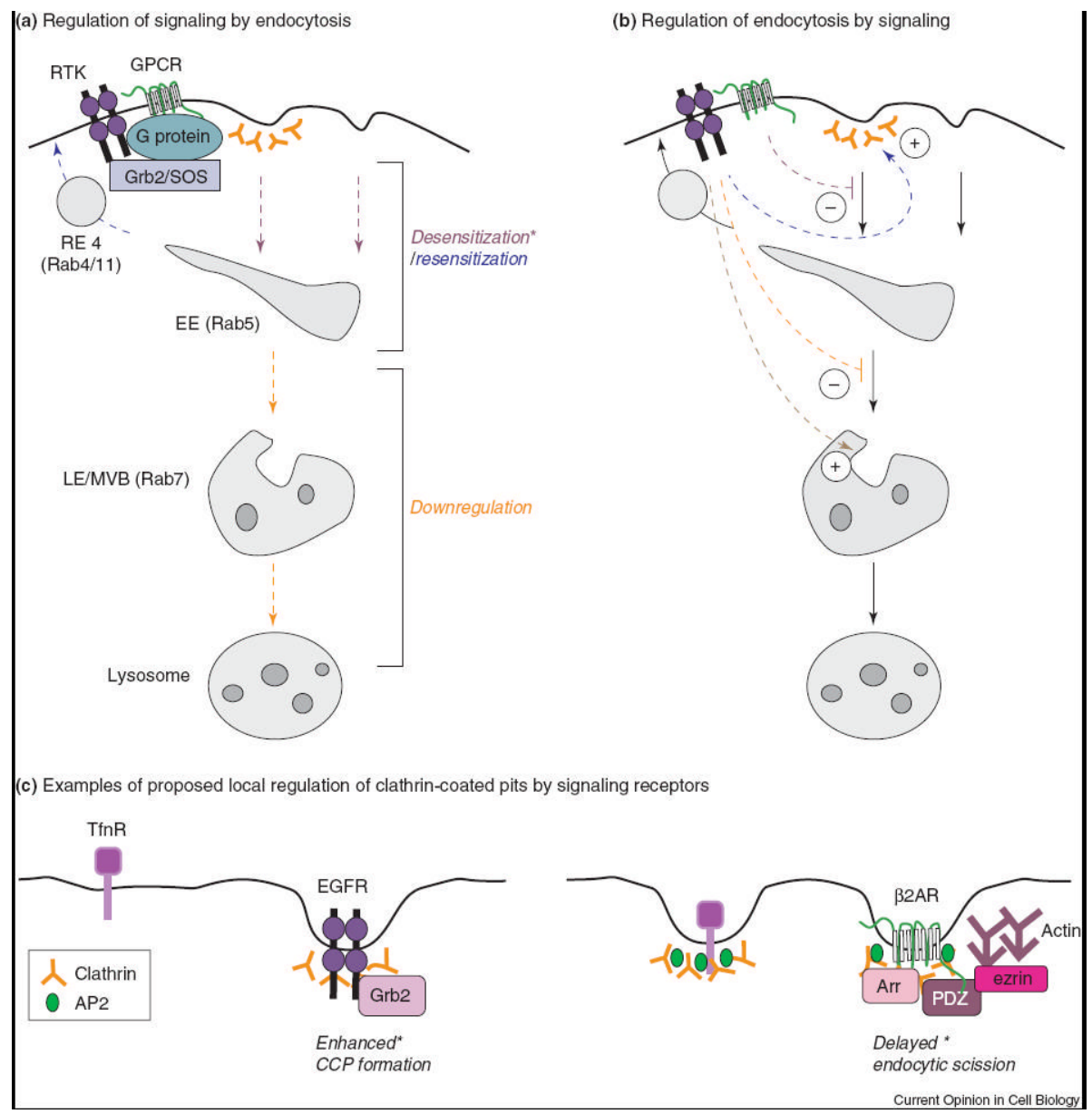

Figure 1. Regulation of plasma membrane signaling by endocytosis and vice versa A, Major regulatory effects of endocytosis on receptor-mediated signaling from the plasma membrane, using an RTK such as the EGF receptor (EGFR) and conventional 7TMR such as $\beta 2 \mathrm{AR}$ (GPCR) as examples. Regulated endocytosis via clathrin -dependent or -independent mechanisms removes receptors from accessibility to plasma membrane-associated signaling pathways, linked by a scaffold protein such as Grb2 or a heterotrimeric G protein, contributing to rapid attenuation or "desensitization" of cell signaling (violet arrows). Recycling to the plasma membrane can reverse this effect, restoring or "resensitizing" cellular responsiveness (blue arrow). Sorting of internalized receptors in MVB and fusion with lysosomes removes receptors from access to cytoplasmic effectors and promotes their proteolysis, resulting in receptor "downregulation" (orange arrows). B, Examples of several proposed regulatory loops by which activated signaling receptors regulate the endocytic pathway, as discussed and cited in the text. The EGF receptor tyrosine kinase is reported to promote coated pit formation under certain conditions (dotted blue line), inhibit early to late endosome maturation (dotted orange line) and promote involution of MVB (dotted brown line). Certain GPCRs, such as the B2AR, reduce the rate of endocytic scission of receptor-containing coated pits (dotted violet line). C, Two recent examples of local regulation of clathrin-coated pits by activated signaling receptors. Left panel: Activated EGFRs, by a mechanism dependent on Grb2, can promote de novo clathrin coated pit formation in cells depleted of AP-2. Such AP-2-deficient coated pits concentrate activated EGFRs but not transferrin receptors (TfnRs). Right panel: GPCRs such as the B2AR concentrate non-uniformly in clathrin-coated pits in association with the regulated adaptor $\beta$-arrestin (arrestin 2 or 3 ). The surface lifetime specifically of GPCR-containing coated 
pits is prolonged by a mechanism that requires PDZ domain mediated binding to scaffold proteins such as EBP50/NHERF1 and linkage to cortical actin via ezrin, whereas coated pits not containing concentrated GPCRs undergo endocytic scission at an unaltered rate. 


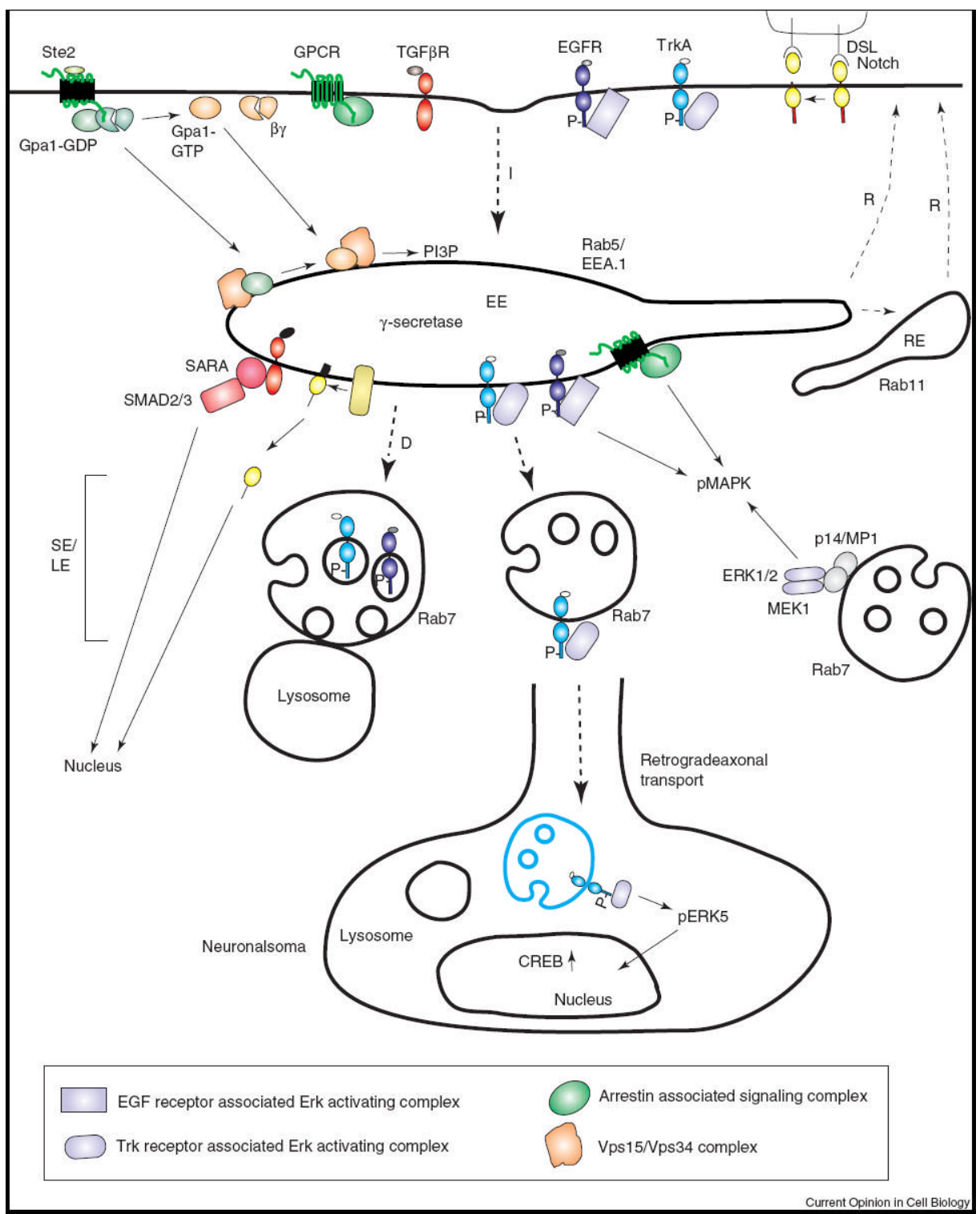

Figure 2. Composite diagram depicting some leading models of endosome-based signal transduction

The figure depicts several types of signaling thought to occur from endosomes, as discussed in the text. Distinct systems are superimposed on the minimum number of endosomal compartments consistent with the presently available data. The figure is an amalgam of data from a number of systems and cell types; thus, only a subset of the indicated signaling events would be expected to occur in any particular cell type. Ligand-induced dimerization of EGF (EGFR) Trk, TGF $\beta$ (TGF $\beta$ R) and Notch is not shown to reduce complexity of the figure. I, internalization; R, recycling; D, degradation; EE, early endosomes; SE/LE, sorting/late endosomes. Endocytic and signaling pathways are shown as broken and solid lines, respectively. 